\title{
Reduced-Complexity Turbo Equalization for High-Density Magnetic Recording Systems
}

\author{
Pornchai Supnithi, Member, IEEE, Renato Lopes, Member, IEEE, and Steven W. McLaughlin, Senior Member, IEEE
}

\begin{abstract}
This paper considers a decision-feedback-equalizer (DFE)-based soft decision detector as an alternative for the Bahl-Cocke-Jelinek-Raviv (BCJR) front-end for a coded magnetic recording channel. In previous work, a bidirectional-arbitrated DFE (BAD) was shown to perform in between a BCJR detector and a minimum mean-square error DFE. We propose a soft-output BAD (S-BAD) which takes advantage of the original structure and is suitable for iterative decoding when used with outer codes. We show that with a convolutional code as an outer code, S-BAD performs close to higher complexity detectors.
\end{abstract}

Index Terms-Bidirectional-arbitrated decision feedback equalizer (BAD), decision-feedback equalizer (DFE), magnetic recording channels, MEEPR4, turbo equalization.

\section{INTRODUCTION}

$\mathbf{T}$ URBO equalization [3] is an effective detection technique for encoded systems that introduce intersymbol interference (ISI), such as magnetic recording channels. A key to the good performance and reasonable complexity of turbo equalization is the fact that the equalizer computes the a posteriori probabilities of the transmitted symbol considering only the channel structure, and using the decoder output as a priori information on the transmitted symbols. This is done exactly by the Bahl-Cocke-Jelinek-Raviv (BCJR) equalizer [1], which is traditionally used for turbo equalization. The complexity of the BCJR equalizer, however, increases exponentially with the channel memory. Many practical implementations require a reduced complexity alternative to the BCJR equalizer.

An important class of reduced-complexity alternatives to the BCJR equalizer consists of a combination of a linear filter and an interference canceller, see [4] and [5] and references therein. In these structures, the received signal goes through a linear filter, and the residual ISI of the filter output is cancelled based on the decoder output. The linear filter may consist of an exact or approximate minimum mean-square error (MMSE) filter [4] or a matched filter [5].

Linear filtering, however, may not be the best ISI-mitigating strategy. In fact, it is known that an MMSE decision-feedback equalizer (DFE) may outperform an MMSE linear equalizer by several decibels [6]. Recently, a bidirectional-arbitrated DFE (BAD) [2] was proposed, and shown to outperform an

Manuscript received December 12, 2002.

P. Supnithi is with the Telecommunications Engineering Department, Faculty of Engineering, King Mongkut's Institute of Technology Ladkrabang, Bangkok 10520, Thailand (e-mail: pornchai@telecom.kmitl.ac.th).

R. Lopes and S. W. McLaughlin are with the School of Electrical and Computer Engineering, Georgia Institute of Technology, Atlanta, GA 30332 USA (rlopes@ece.gatech.edu; swm@ece.gatech.edu).

Digital Object Identifier 10.1109/TMAG.2003.816487
MMSE-DFE. In this paper, we propose a soft-output BAD (S-BAD) that produces extrinsic information and that can benefit from the information provided by the outer code. It consists of a forward and reverse MMSE-DFE, where the residual ISI is cancelled based on the decoder and equalizer outputs. The outputs of the DFEs are combined to form the soft outputs. We will show that, when used in a turbo equalizer, the S-BAD performs close to the BCJR equalizer and is comparable to the turbo-decision-aided equalizer (DAE) proposed in [5]. However, the S-BAD is less complex than both the BCJR equalizer and the turbo-DAE, which requires a use of BCJR equalizer for the first iteration.

\section{Turbo EQUalization Using S-BAD}

\section{A. $B A D$}

The BAD [2] uses two MMSE-DFEs to recover channel inputs in a forward and reverse manner as shown in Fig. 1. Given the input $x,\left(\left\{x_{k}\right\}, k=0, \ldots, M-1\right)$, and the channel impulse response $\left\{h_{k}\right\}, k=-L_{2}, \ldots, L_{1}$, the received sequence $\boldsymbol{r},\left(\left\{r_{k}\right\}, k=0, \ldots, N-1\right)$ can be expressed as

$$
r_{k}=\sum_{i=-L_{2}}^{L_{1}} x_{k-i} h_{i}+n_{k}
$$

where $n_{k}$ is an additive white noise with variance $\sigma^{2}$.

Consider the top MMSE-DFE which consists of two filters: a feedforward filter $\mathbf{F}=\left\{f_{k}\right\},\left\{k=0, \ldots, N_{f}\right\}$ of length $N_{f}+1$, and a strictly causal feedback filter $\mathbf{B}=\left\{b_{k}\right\},\{k=$ $\left.1, \ldots, N_{b}\right\}$ of length $N_{b}$. The coefficients are derived under the MMSE criterion [6]. The equalizer output is

$$
z_{k, f}=\sum_{j=0}^{N_{f}} f_{j} r_{k-j}+\sum_{j=1}^{N b} b_{j} \hat{x}_{k-j, f} .
$$

The decided channel bit at time $k, \hat{x}_{k, f}$ is obtained from slicing $z_{k, f}$. Both MMSE-DFEs are independently designed; they differ for asymmetric channels. The lengths $N_{f}$ and $N_{b}$ depend largely on whether the channels are minimum, mixed, or maximum phase and on the lengths $L_{1}$ and $L_{2}$ [6]. For the bottom MMSE-DFE, the feedforward filter $\mathbf{F}_{\mathbf{R}}$ and feedback filter $\mathbf{B}_{\mathbf{R}}$ are designed for the time-reversed channel $\tilde{h}_{k}$. Thus, we see that the top MMSE-DFE cancels precursor ISI, while the bottom MMSE-DFE cancels postcursor ISI.

The BAD algorithm implements forward and reverse MMSE DFEs to obtain two channel input estimates: a forward estimate $\hat{x}_{k, f}$ and a reverse one $\hat{x}_{k, b}$. In order to compute the Euclidean distance of the received sequence, we first obtain their estimates 


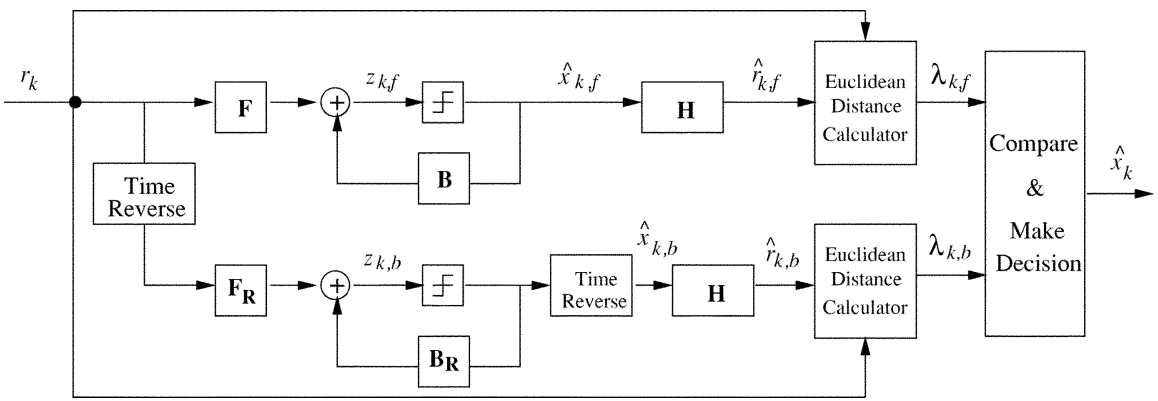

Fig. 1. Schematic of a bidirectional DFE.

based on $\hat{x}_{k, f}$ and $\hat{x}_{k, b}$. The estimate of received sequence $\boldsymbol{r}$ can be computed from

$$
\begin{aligned}
& \hat{r}_{k, f}=\sum_{i=-L_{1}}^{L_{2}} h_{i} \hat{x}_{k-i, f} \\
& \hat{r}_{k, b}=\sum_{i=-L_{1}}^{L_{2}} h_{i} \hat{x}_{k-i, b} .
\end{aligned}
$$

An arbitration rule then decides on each bit based on the least Euclidean distance within the window size $W_{1}+W_{2}+1$. It calculates the parameter $\lambda_{k, f}$ and $\lambda_{k, b}$ given by

$$
\begin{aligned}
\lambda_{k, f} & =\sum_{n=-W_{1}}^{W_{2}}\left|r_{k+n}-\hat{r}_{k+n, f}\right|^{2} \\
\lambda_{k, b} & =\sum_{n=-W_{1}}^{W_{2}}\left|r_{k+n}-\hat{r}_{k+n, f}\right|^{2} .
\end{aligned}
$$

The decision $\hat{x}_{k}$ is $\hat{x}_{k, f}$ if $\lambda_{k, f}<\lambda_{k, b}$ and $\hat{x}_{k, b}$ otherwise.

\section{B. Extrinsic Information Calculation}

An S-BAD needs to produce extrinsic information of channel bits, which is defined as

$$
\begin{aligned}
L_{e x t}\left(x_{k}\right) & =\ln \left\{\frac{P\left(\boldsymbol{r} \mid x_{k}=1\right)}{P\left(\boldsymbol{r} \mid x_{k}=-1\right)}\right\} \\
& =\ln \left\{\frac{\sum_{\mathbf{C}_{\mathbf{i}} \in S^{+1}} P\left(\boldsymbol{r} \mid \mathbf{C}_{\mathbf{i}}\right)}{\sum_{\mathbf{C}_{\mathbf{i}} \in S^{-1}} P\left(\boldsymbol{r} \mid \mathbf{C}_{\mathbf{i}}\right)}\right\} \\
& =\ln \left\{\frac{\sum_{\mathbf{C}_{\mathbf{i}} \in S^{+1}} e^{-\frac{\left\|\boldsymbol{r}-\mathbf{C}_{\mathbf{i}}\right\|^{2}}{2 \sigma^{2}}}}{\sum_{\mathbf{C}_{\mathbf{i}} \in S^{-1}} e^{-\frac{\left\|\boldsymbol{r}-\mathbf{C}_{\mathbf{i}}\right\|^{2}}{2 \sigma^{2}}}}\right\}
\end{aligned}
$$

where $S^{+1}$ and $S^{-1}$ are sets of codeword $\mathbf{C}_{\mathbf{i}}$ with bit $x_{k}=1$ and -1 , respectively. We simplify $L_{e x t}\left(x_{k}\right)$ based on two cases.

Case 1) $\hat{x}_{k, f} \neq \hat{x}_{k, b}$

Assume that $\hat{x}_{k, f}=1$ and $\hat{x}_{k, b}=-1$. In this case, we can simplify (9) by considering only one member of $S^{+1}$ and one member of $S^{-1}$ : respectively $\hat{r}_{k, f}$ and $\hat{r}_{k, b}$. Using only values inside the arbitration window is similar to approximating the summation in (9) with the maximum term, and yields

$$
L_{e x t}\left(x_{k}\right)=\ln \left\{\frac{e^{-\frac{\lambda_{k, f}}{2 \sigma^{2}}}}{e^{-\frac{\lambda_{k, b}}{2 \sigma^{2}}}}\right\}^{\hat{x}_{k, f}}=\hat{x}_{k, f} \frac{\left(\lambda_{k, b}-\lambda_{k, f}\right)}{2 \sigma^{2}} \text {. }
$$

The expression is the same if we assume that $\hat{x}_{k, f}=-1$ and $\hat{x}_{k, b}=1$. The extrinsic information, $L_{e x t}\left(x_{k}\right)$, can therefore be computed directly from existing parameters, $\lambda_{k, f}$ and $\lambda_{k, b}$.

Case 2) $\hat{x}_{k, f}=\hat{x}_{k, b}$

In this case, the output of the forward and reverse MMSE-DFE belong to the same set $\left(S^{+1}\right.$ or $\left.S^{-1}\right)$, so a different approach must be followed. As in [7], we assume that $z_{k, f}=A_{i} x_{k}+$ noise of variance $\sigma^{2}$. Using the output of both equalizers, this yields

$$
L_{e x t}\left(x_{k}\right)=\frac{2 A_{i}\left(z_{k, f}+z_{k, b}\right)}{\sigma^{2}} .
$$

In summary,

$$
L_{e x t}\left(x_{k}\right)= \begin{cases}\frac{\hat{x}_{k, f}\left(\lambda_{k, b}-\lambda_{k, f}\right)}{2 \sigma^{2}}, & \text { if } \hat{x}_{k, f} \neq \hat{x}_{k, b} \\ \frac{2 A_{i}\left(z_{k, f}+z_{k, b}\right)}{\sigma^{2}}, & \text { otherwise. }\end{cases}
$$

\section{Threshold Adjustment}

To implement an iterative decoding process, the outer decoder such as turbo decoder passes a priori information of channel bit $x_{k}, L\left(x_{k}\right)$, to the S-BAD equalizer. This information can be used to adapt the threshold of the slicer of each bit, $\tau_{k}$. Let $\left\{x_{k}\right\}$ be nonequiprobable binary channel bits, i.e., $P\left(x_{k}=1\right) \neq$ $P\left(x_{k}=-1\right)$, the decision rule for additive white Gaussian noise (AWGN) is to decide $\hat{x}_{k}=1$ if the received bit is greater than $\tau_{k}$, otherwise decide $\hat{x}_{k}=-1$ [6]. The threshold $\tau_{k}$ is given by

$$
\tau_{k}=\frac{\sigma_{n}^{2}}{2} \ln \frac{P\left(x_{k}=-1\right)}{P\left(x_{k}=1\right)}=-\frac{\sigma_{n}^{2}}{2} L\left(x_{k}\right) .
$$

For a case of equiprobable signaling, $\tau_{k}=0$.

\section{Independence Criterion}

An important property to ensure iterative gain is that the extrinsic information $L_{e x t}\left(x_{k}\right)$ is independent of $L\left(x_{k}\right)$ [4] or, equivalently, in this case, $\hat{x}_{k, f}$ and $\hat{x}_{k, b}$. In Section II-B, when $\hat{x}_{k, f}=\hat{x}_{k, b}$, we pass $2 A_{i}\left(z_{k, f}+z_{k, b}\right) / \sigma^{2}$ to the outer decoder; it is clear that because $z_{k, f}$ and $z_{k, b}$ are both independent of $\hat{x}_{k, f}$ and $\hat{x}_{k, b}$ from (2), they are independent of $L\left(x_{k}\right)$. In the case when $\hat{x}_{k, f} \neq \hat{x}_{k, b}, L_{e x t}\left(x_{k}\right)=\hat{x}_{k, f}\left(\lambda_{k, b}-\lambda_{k, f}\right) / 2 \sigma^{2}$. After some manipulation, $L_{\text {ext }}\left(x_{k}\right)$ can be expressed as a function of two variables, i.e.,

$$
L_{e x t}\left(x_{k}\right)=f\left(\hat{x}_{k, f}+\hat{x}_{k, b}, \hat{x}_{k, f}\left(\hat{x}_{k, f}-\hat{x}_{k, b}\right)\right) .
$$




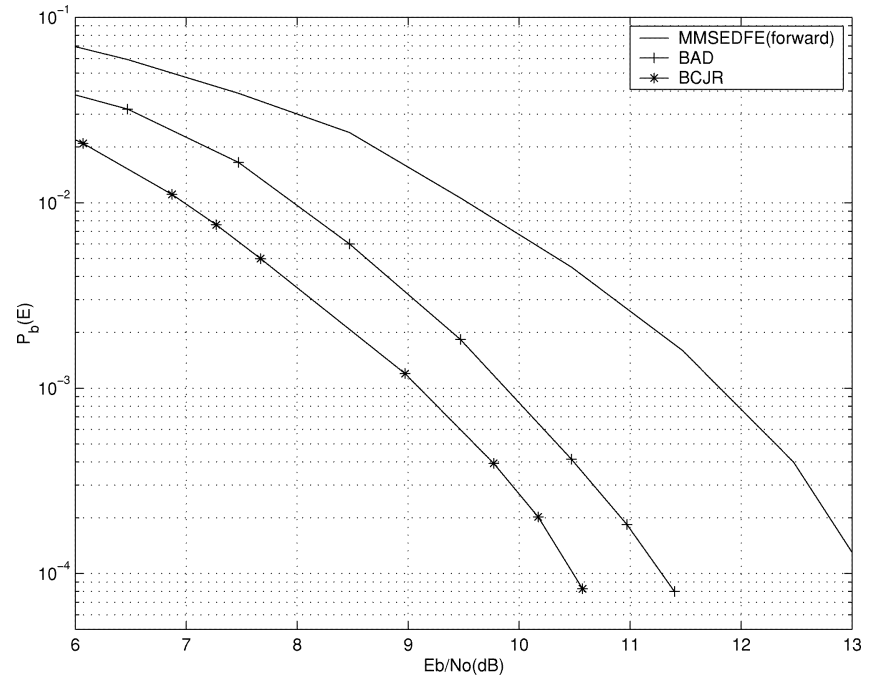

Fig. 2. BAD performance versus BCJR on MEEPR4 channel.

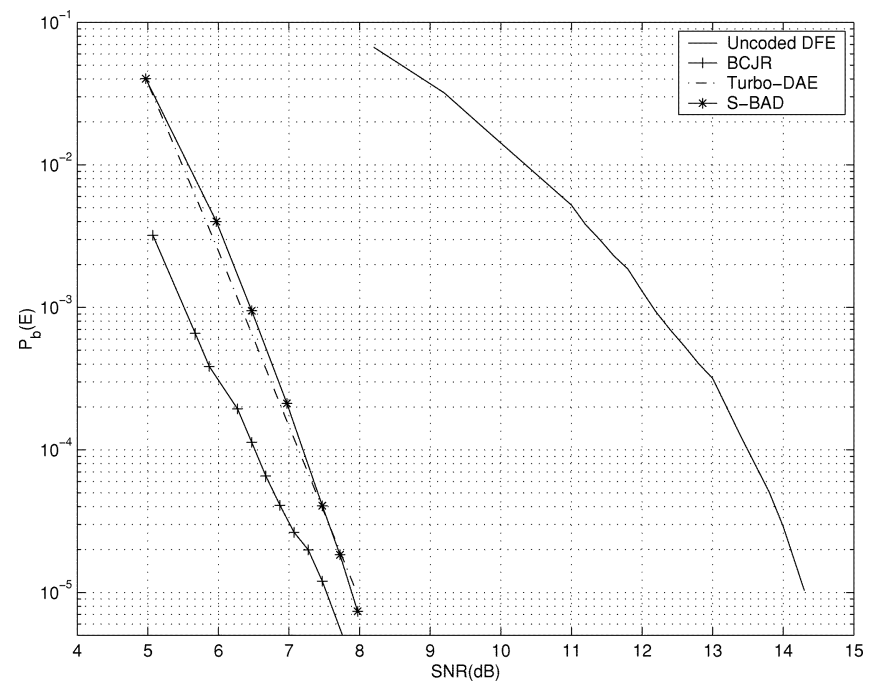

Fig. 3. Performance of SCCC with $R=8 / 9$ and with BCJR, S-BAD, and Turbo-DAE on MEEPR4 channel after eight iterations.

Since $\hat{x}_{k, f} \neq \hat{x}_{k, b}$, i.e., $\hat{x}_{k, f}=-\hat{x}_{k, b}$ for binary inputs, the term $\hat{x}_{k, f}+\hat{x}_{k, b}=0$ and $\hat{x}_{k, f}\left(\hat{x}_{k, f}-\hat{x}_{k, b}\right)=2$. Thus $L_{e x t}\left(x_{k}\right)$ is independent of $\hat{x}_{k, f}$ and $\hat{x}_{k, b}$, and equivalently, $L\left(x_{k}\right)$.

\section{Performance Results}

We first illustrate the performance of BAD on an uncoded MEEPR4 channel $H(D)=5+4 D-3 D^{2}-4 D^{3}-2 D^{4}$ with i.i.d. binary channel inputs. The tap coefficients $N_{f}$ and $N_{b}$ are selected to be 14 and 9 , respectively. For simplicity, we select $W=W_{1}=W_{2}=15$. The BAD performance compared with MMSE-DFE and BCJR equalizer is shown in Fig. 2. An MMSE-DFE loses about $2.6 \mathrm{~dB}$ from the MAP equalizer at $P_{b}(E)=1.1 \times 10^{-4}$. With $\mathrm{BAD}$, however, the loss is reduced to less than $1 \mathrm{~dB}$. Thus, BAD is a potential candidate as a hard-decision equalizer. In Fig. 3, we consider S-BAD when used with a serially-concatenated convolutional code (SCCC). The outer code is a rate- $1 / 2$ convolutional code with $\left(g_{1}, g_{2}\right)=(31,27)_{o c t}$, punctured to form a rate-8/9 code. The MEEPR4 channel is treated as an inner code. The block size is $N=4558$. We define $S N R=E_{b} / N_{o}$, where $E_{s}=R E_{b} * \sum_{k}\left|h_{k}\right|^{2}$, and noise variance $\sigma^{2}=N_{0} / 2$. Because at early iterations, the a priori information sent from the outer decoder can be unreliable, we introduce a weight factor on the threshold, i.e., $\tau_{k}=-w_{i}\left(\sigma_{n}^{2} / 2\right) L\left(x_{k}\right)$, where $w_{i}$ is a weight factor at iteration $i$. In the simulation, the weight factor $\mathbf{W}=[0.2,0.3,0.35,0.4,0.5,0.55,0.6,0.7]$ is selected empirically to provide lowest bit error rate for this channel. We find that the gain of the forward and reverse MMSE-DFE $A_{f, i}$ and $A_{b, i}$ center around 0.5 , thus we assume them to be constant. The system with channel BCJR is superior to Turbo-DAE and $\mathrm{S}-\mathrm{BAD}$ as shown in Fig. 3. The S-BAD performance is comparable to Turbo-DAE and is able to surpass it at $P_{b}(E)=10^{-5}$ by $\approx 0.1 \mathrm{~dB}$. It also reduces the decoding complexity as it does not require the use of BCJR decoder at the first iteration like Turbo-DAE.

\section{CONCLUSION}

In this paper, a new soft DFE-based equalizer based on BAD (S-BAD) has been proposed. It benefits from the better performance of BAD when compared to linear equalizers and the traditional DFE, while having low computational complexity. When used with an outer code, iterative decoding can be accomplished by using the decoder output to update the threshold of both MMSE-DFE slicers. On the MEEPR4 channel, the performance of S-BAD is clearly similar to that of Turbo-DAE, and close to that of the BCJR detector. An advantage of S-BAD is complexity reduction because it does not require the use of the BCJR equalizer at any iteration. Even for one iteration, S-BAD performs better than MMSE-DFE. The S-BAD is also applicable to other environments such as wireless and optical communications provided that the channel coefficients are known. In practice, the channel needs to be estimated.

\section{REFERENCES}

[1] L. Bahl, J. Cocke, F. Jelinek, and J. Raviv, "Optimal decoding of linear codes for minimizing symbol error rate," IEEE Trans. Inform. Theory, vol. IT-20, pp. 284-287, Mar. 1974.

[2] C. McGahey, A. Singer, and U. Madhow, "BAD: A bi-directional arbitrated decision feedback equalizer," presented at the 34th Conf. Information Sciences and Systems, Princeton, NJ, Mar. 15-17, 2000.

[3] C. Douillard, M. Jezequel, C. Berrou, A. Picart, P. Didier, and A. Glavieux, "Iterative correction of intersymbol interference: turbo-equalization," Eur. Trans. Telecomm., vol. 6, no. 5, pp. 507-511, Sept. 1995

[4] M. Tuchler, R. Koetter, and A. Singer, "Turbo equalization: principles and new results," IEEE Trans. Commun., vol. 50, pp. 754-767, May 2002.

[5] Z. Wu and J. M. Cioffi, "Low-complexity iterative decoding with decision-aided equalization for magnetic recording channels," IEEE J. Select. Areas Commun., vol. 19, pp. 699-708, Apr. 2001.

[6] J. Proakis, Digital Communications, 3rd ed. New York: McGraw-Hill, 1995.

[7] R. Lopes and J. Barry, "Blind iterative channel identification and equalization,” in Proc. ICC'01, Helsinki, Finland, 2001, pp. 2256-2260. 